\title{
RECOPILACIÓN DE EXPERIENCIAS EN LA POTABILIZACIÓN DEL AGUA POR MEDIO DEL USO DE FILTROS
}

\author{
COMPILATION OF EXPERIENCES AGAINST THE WATER PURIFICATION \\ THROUGH THE USE OF FILTERS \\ RECOLHA DE EXPERIÊNCIAS NA POTABILIZACÃO DO ÁGUA \\ POR MÉDIO DO USO DE FILTROS
}

Juan David Córdoba Paradaํ, Rocío Acosta Alarcón², Juan Fernando Pacheco³ ${ }^{3}$ Catalina Ramírez ${ }^{4}$

Fecha de recibido: febrero 01 de 2016 | Fecha de aprobado: marzo 27 de 2016

\section{Resumen}

Este documento expone una revisión bibliográfica de casos de estudio que evidencian la importancia e implementación de los filtros como alternativa para la potabilización del agua. Se presentan cinco casos aplicados en Colombia donde se utilizaron filtros potabilizadores de agua -filtros de arena, arcilla, velas de cerámica y ultrafiltración, entre otros- en comunidades vulnerables; y dos casos internacionales que destacan la importancia que cumplen los filtros en las comunidades rurales en donde en ocasiones, el acceso al agua potable es nulo - los filtros utilizados fueron de arena y cerámica con plata coloidal-. Se evidenció que el uso de filtros para la potabilización de agua en las comunidades vulnerables tiene buena aceptación y replicación puesto que el acceso al agua segura, en estos casos, es limitado o no existe.

Palabras clave: agua potable, recurso hídrico, filtración, comunidades vulnerables.

1 Estudiante de ingeniería en Agroecología, integrante de Ingenieros sin Fronteras Colombia - ISF-COL -, jdcpras@hotmail.com.

2 Ingeniera en Agroecología, máster en Ingeniería Ambiental, grupo de investigación Desarrollo Regional M.D, roacosta@uniminuto.edu.

3 Ingeniero Industrial, gerente Parque Científico de Innovación Social, grupo de investigación Desarrollo Regional, M.D, jpacheco@uniminuto.edu.

4 Ingeniera Industrial, docente de la Universidad de los Andes e integrante de Ingenieros sin Fronteras Colombia-ISF-COL-, mariaram@uniandes.edu.co. 


\section{Abstract}

This article exposes a bibliographic review of cases of study that evidence the importance of the implementation of filters as an alternative for the purification of the water. Five cases applied in Colombia are presented where water purification filters were used -sand filters, clay, ceramic candles and ultrafiltration, among others - in vulnerable communities and two international cases that emphasize the importance of the filters inside the rural communities, where the access to drinkable water can be null sometimes -the used filters were made of sand and ceramic with colloidal silver-. It was evidenced that the use of filters to purify water in the vulnerable communities has a good acceptance and replication given that the access to drinkable water, in these cases, is limited or null.

Keywords: drinkable water, hydric resource, filtration, vulnerable communities.

\section{Resumo}

Este documento expõe uma revisão bibliográfica de casos de estudo que evidencian a importância e implementação dos filtros como alternativa para a potabilização do água. Apresentam-se cinco casos aplicados em Colômbia onde se utilizaram filtros potabilizadores de água - filtros de areia, arcilla, vá-as de cerâmica e ultrafiltra, entre outros - em comunidades vulneráveis; e dois casos internacionais que destacam a importância que cumprem os filtros nas comunidades rurais em onde em ocasiões, o acesso à água potável é nulo -os filtros utilizados foram de areia e cerâmica com prata coloidal-. Se evidenció que o uso de filtros para a potabilização de água nas comunidades vulneráveis tem boa aceitação e replicação já que o acesso à água segura, nestes casos, é limitado ou não existe.

Palavras-chave: água potável, recurso hídrico, filtragem, comunidades vulneráveis. 


\section{INTRODUCCIÓN}

La presente revisión está encaminada a recopilar información sobre los filtros como estrategia de potabilización del agua. La información obtenida versa sobre soluciones alternativas enfocadas al desarrollo de las comunidades rurales y urbanas a partir del suministro y calidad del agua para mitigar los problemas que se presentan por el consumo de agua inapropiada - no potable- que, en ocasiones, puede causar muerte por enfermedades diarreicas agudas -EDAS-.

El consumo de agua potable se ha incrementado rápidamente teniendo en cuenta que sólo el $0.01 \%$ del agua existente en la Tierra se puede usar para las actividades humanas -consumo directo, industria y agricultura-; el excedente se encuentra en los océanos $-97 \%$ - y en forma de nieve o de hielo $-2.99 \%$ - (Giordan \& Souchon, 1995). Al menos 1.000 millones de personas no cuentan con acceso a agua potable y un $20 \%$ de la población total no dispone de agua (onU, 2000).

Masari (2003) menciona que la cantidad de agua que tenemos en la Tierra no aumenta ni disminuye pero la población humana sí ha crecido y con ella la necesidad que tiene de este recurso, además indica que el agua apta para el ser humano ha disminuido en gran medida, lo que nos llevaría a enfrentar una crisis global, evidentemente en torno al agua, en el siglo Xxı. Desde la Unesco se prevé que en 2030 la Tierra tendrá que enfrentarse a una falta mundial del $40 \%$ de agua en un escenario climático donde no haya un cambio en las actividades industriales, mineras y agrícolas convencionales, por lo que es fundamental que tomemos conciencia en el uso y consumo responsable del agua. (Júarez, 2016).

En la Declaración Universal de Derechos Humanos de 1948 se hace constar que toda persona tiene derecho a un nivel de vida digno para asegurar su salud, su bienestar y el de su familia, lo que incluye el derecho al agua potable (Defensoría del Pueblo, 2006). Por lo anterior, la disminución de la calidad del agua se ha convertido en motivo de intranquilidad a nivel mundial dado el crecimiento exponencial de la población humana y la expansión de la industria, la agricultura y el cambio climático, causantes de alteraciones en el ciclo hidrológico (Navarro, 2008). El principal problema relacionado con la calidad del agua lo constituye la eutrofización, el cual es el resultado del aumento de los niveles de nutrientes, generalmente fósforo y nitrógeno (onU, 2015).

Según Cavero (2004) y la Universidad de Manizales (2015), para darle respuesta al problema de la falta de agua potable a nivel mundial se han planteado diferentes estrategias de manejo y aplicación de filtros potabilizadores de agua. Ésta es una medida de acción a la problemática actual que se presenta por los desastres naturales, el cambio climático y las zonas de conflicto, donde el agua no tiene las mejores cualidades para el consumo humano. A su vez, se debe tener en cuenta que existen comunidades rurales que no cuentan con plantas de tratamiento de agua o tienen acueductos veredales ineficientes.

De acuerdo a lo anterior, es importante resaltar que existen métodos que se encuentran al alcance de todos y pueden proporcionar una mejor calidad del recurso hídrico a las comunidades que lo necesitan. Estos métodos se basan en el principio de la filtración, un proceso en el que se retiene, por un medio poroso, la materia en suspensión contenida en un fluido (García, 1995). En la actualidad se han desarrollado diferentes métodos para realizar la filtración y potabilización de agua: filtro de carbón activado, filtro de tela, filtro lento de arena, filtro de velas, filtro de cerámica y filtro de membrana de ultrafiltración (UNAD, 2015).

\section{SISTEMAS DE FILTRACIÓN UTILIZADOS ACTUALMENTE}

\section{Filtro lento de arena}

La filtración por arena es, de las tecnologías para el tratamiento de agua, la más antigua de la que se tenga registro y opera fácilmente teniendo un efluente de buena calidad (Lesikar, 2000). Se utiliza principalmente para eliminar características de turbiedad, aunque por medio de procesos físicos y biológicos puede eliminar patógenos presentes en el agua no apta para el consumo humano. Estos sistemas son de bajo costo porque utilizan materiales locales como las arenas de diferentes tamaños, grava, geotextil y recipientes plásticos ver Tabla 1, sección A- (Perdomo, 2014). 


\section{Filtro de cerámica}

Es un filtro de bajo costo para uso doméstico en comunidades urbanas y rurales que trata el agua contaminada para el consumo directo. Está hecho de una mezcla de arcilla y aserrín -este último elemento es el que da la porosidad-. El filtro es frotado con una solución de plata coloidal y en algunos casos es colocado dentro de un recipiente. Puede ser construido por alfareros locales usando materiales propios de la zona y no requiere de electricidad o tecnología avanzada - ver Tabla 1, secciones B1, B2, B3-. (Salud, 2005).

\section{Filtro de vela}

Es un filtro portátil compuesto por dos baldes de polietileno de alta densidad con una capacidad de 20 litros cada uno. Se ubica un balde encima del otro: el primero contiene una o dos velas de cerámica compuestas por materiales microporosos que permiten la retención de partículas sólidas y microorganismos; el segundo balde pasa el agua filtrada saludable para el consumo (Rojas \& Guevara, 2000). Es una de las más importantes formas de mejoramiento de la calidad del agua a nivel domiciliario, además, ha probado ser muy efectiva y de fácil implementación -ver Tabla 1, sección C-. (Burgos, 2006).

\section{Filtro de carbón}

Este tipo de filtración - con la utilización de carbónpuede ser bastante efectiva para la remoción de algunos sabores, olores y colores. Se puede usar el carbón común disponible localmente o carbón activado que, aunque es más efectivo, puede aumentar los costos. (Organización Mundial de la Salud, 2009).

\section{Filtro de ultrafiltración}

La ultrafiltración utiliza filtros de membrana con tamaños de poros habituales de $1 \mathrm{a} 10 \mathrm{~nm}$, los cuales pueden eliminar partículas tan pequeñas como las macromoléculas de proteínas. La ultrafiltración -UFes una tecnología excelente para asegurar una calidad constante de agua pura con respecto a las partículas, las bacterias y los pirógenos; asimismo, su funcionamiento es por gravedad y no requiere de energía -ver Tabla 1, sección D- (Elga Ovelia, 2016).

\section{METODOLOGÍA}

Esta revisión bibliográfica reúne documentos web en los que se evidencian diferentes casos de estudio de filtros potabilizadores de agua implementados en investigaciones de la Universidad de Manizales, la Universidad Abierta y a Distancia -UNAD-, la Universidad de los Andes, la Universidad de Pamplona, la Universidad de Kassel, entre otras. Se usaron algunos casos expuestos en las memorias del Seminario de intercambio de experiencias, tecnologías y metodologías en agua, saneamiento $e$ higiene en zonas vulnerables y en emergencias, realizado el 15 de octubre del 2014 como parte de la mesa del clúster WASH que lidera Unicef en Colombia. Con la información obtenida se recopiló la Tabla 1. Resumen de caracterización de filtros potabilizadores de agua, que incluye los costos y la eficiencia según los caudales de filtración.

\section{Casos de estudio}

A partir de 15 casos de estudio se expone una recopilación de las siete experiencias de campo más representativas para los diferentes métodos de filtración. El objetivo es presentar las principales características de las experiencias en comunidades donde se han utilizado los filtros potabilizadores de agua y la importancia que representan como método de acceso al recurso; se revisan casos a nivel nacional e internacional en los que se resalta el uso de filtros artesanales e industriales. Se debe tener en cuenta que en algunos casos la información entregada por el investigador no es una razón total por la cual no se pueda establecer homogeneidad para la comparación.

Proyecto Guayabal de Síquima, Ingenieros sin fronteras Colombia -ISF-COL-: Mejoramiento de la calidad del agua de una comunidad del departamento de Cundinamarca, Colombia

Para el desarrollo del proyecto se realizó un diagnóstico en el que se hicieron pruebas de laboratorio para conocer la calidad del agua en aspectos físico-químicos y microbiológicos que demostraron la baja calidad del agua de consumo en la comunidad de Guayabal de Síquima. Esto llevó a un acercamiento entre el grupo de investigación ISF-COL y la comunidad local.

Se realizaron talleres con metodologías de acción participativa para la implementación de filtros 
lentos de arena y se entregaron a la comunidad 14 unidades filtrantes con una capacidad aproximada de 60 litros que beneficiaron 16 familias; luego se realizaron pruebas al agua procesada siguiendo los parámetros físico-químicos y microbiológicos de: turbidez, $\mathrm{pH}$, conductividad, color verdadero, coliformes totales y Escherichia coli. Según los resultados de campo se evidenció que el sistema de filtración implementado eliminó el $90 \%$ de heces y coliformes totales durante el periodo de maduración de la cama de filtrado. Más aún, se mejoraron las condiciones organolépticas del agua después de ser tratada, por lo que se comprobó la disminución de sólidos en suspensión y se permitió el acceso al agua potable para las personas de la comunidad intervenida (Ramírez, et al., 2011).

Purificación de agua por medio de filtros lentos de arena en la comunidad de Kuychiro-Cusco, Perú

Esta investigación tuvo como objetivo general purificar el agua del rio Kuychiro para consumo humano mediante la filtración lenta de arena. El procesoincluyóla elaboración del filtro con materiales tales como el difusor, la válvula flotadora, geotextil, tubería de drenaje, recipiente y arena de diferentes diámetros. El estudio de la implementación de los sistemas de filtración y el análisis del agua dieron como resultado que las muestras cumplieron con las especificaciones técnicas para el consumo humano.

Se concluyó que la utilización de filtros de arena en la purificación del agua para consumo humano es factible dado que los niveles en remoción de coliformes totales bajaron un $80.91 \%$, y un $67.39 \%$ en coliformes termotolerantes. Además, demostró ser una tecnología de fácil acceso, manejo y mantenimiento para personas de escasos recursos, teniendo un caudal promedio de $2 \mathrm{~L}$ por hora aproximadamente (Barrientos Echegaray, Tello, Tito \& Palomino, 2009).

Proyecto: Fundación Acción contra el Hambre ACF- en Nariño, Córdoba y Putumayo, Colombia

ACF es una ONG internacional que busca mejorar y asegurar una nutrición sana tanto en comunidades vulnerables como en los temas referentes a sanidad y calidad de agua e instalaciones. El aporte de la
ONG referente al tema de agua y saneamiento se fundamentó en el entrenamiento, apropiación y aplicación de herramientas para el manejo del recurso hídrico, usando como estrategia la entrega masiva de filtros de velas potabilizadores de agua, tanques de almacenamiento y la participación en procesos de investigación en alianza con la Universidad de Boyacá (RECA, WASH Cluster, Cruz Roja, Unicef, 2014).

Por lo anterior, se realizó un estudio comparativo en los laboratorios de la Universidad de Boyacá en Tunja sobre el proceso de filtración, entre una y dos velas cerámicas, en el que se concluyó que se había reducido la turbiedad a valores inferiores al 2.0 UNT durante el tiempo de operación en ambos sistemas de filtración, lo cual dio cumplimiento al valor máximo de turbiedad reglamentado por la Resolución 2115 de 2007 para agua potable de Colombia. Respecto al número de velas, se alcanzaron eficiencias de filtración similares entre una y dos, aparte de la remoción de microorganismos como el Escherichia coli y coliformes totales. Las diferencias se concentraron en aspectos económicos, operativos y de mantenimiento al momento de remplazar las velas filtrantes; sin embargo, el filtro de dos velas mostró mayor eficiencia en el caudal de filtración. Se obtuvo una remoción de patogenos de un $99 \%$ y un caudal de filrtracion de $1 \mathrm{~L}$ por hora aproximadamente (Pérez Vidal, Díaz \& González, 2014).

\section{Estudio de alternativas tecnológicas para el} tratamiento básico del agua lluvia de uso doméstico en el consejo comunitario de la comunidad negra de Los Lagos, Buenaventura, Colombia

Este caso resalta la importancia de recolectar aguas lluvias en las zonas de alta precipitación. Por lo anterior, se implementaron sistemas de canales y tanques de almacenamiento para la recolección del líquido, el cual posteriormente se sometió a un proceso de filtración de velas. Esta tecnología consta de dos velas de cerámica y dos baldes de polietileno de 20 litros que se colocan uno encima del otro: el de la parte superior contiene las velas cerámicas -medio filtrante- y el inferior es el encargado de almacenar el agua tratada, lista para el consumo directo de las personas. La comunidad fue capacitada para el uso y aprovechamiento de estos sistemas (Salud, 2005). 
Intervención de la Fundación para la asistencia humana en emergencias y desarrollo urbano y rural Sahed en comunidades de Córdoba, Colombia

Sahed es una ong que trabaja para responder ante situaciones de emergencia; desarrolla y fortalece las capacidades técnicas y sociales de instituciones públicas, privadas y comunitarias. Esta organización intervino en el departamento de Córdoba y el alto Sinú en comunidades con altos grados de vulnerabilidad en cuanto suministro y calidad del agua; allí se realizó una caracterización de la zona y un acercamiento entre la organización y la comunidad para dar inicio a capacitaciones referentes al manejo y mantenimiento del filtro potabilizador de agua Ekofil -ver Tabla.1, sección B1-.

Ekofil es un filtro de agua portátil utilizado por Sahed, práctico, de fácil instalación y manejo, y su funcionamiento no requiere electricidad ni especialistas para operarlo. Está elaborado con arcilla porosa tratada con un baño de plata coloidal y trabaja por la acción de la gravedad, generando agua limpia, fresca y libre de bacterias (Filtro de arcilla Ekofil, 2015). En el estudio se identificaron las bondades y cualidades del manejo del filtro dado que se logró mejorar la calidad de vida y el suministro de agua potable en las comunidades intervenidas de Tierralta - Córdoba - e indígenas del alto Sinú. Lo anterior comprobó la eficiencia en la remoción de partículas patógenas microbiológicas y de sedimentación en un 99\% con caudal promedio de filtración de $1 \mathrm{~L}$ por hora aproximadamente (RECA, et al., 2014).

Prueba de aceptación del filtro de cerámica impregnado con plata coloidal en el barrio $\mathrm{El}$ Ocotal de Guinope, Honduras

Se identificaron grandes trazas de coliformes totales y fecales en el agua de la comunidad del barrio El Ocotal de Guinope, por lo que se implementó un filtro de cerámica con una capa de plata coloidal. Se trabajó con la comunidad poniendo a prueba la eficiencia del filtro mediante el análisis de muestras compuestas, analizadas antes y después de la filtración. El filtro resultó ser muy eficiente en la descontaminación microbiológica, ya que redujo a cero las unidades formadoras de colonias en una muestra compuesta, una disminución notable de turbiedad. No se encontró cloro libre en las muestras de agua filtrada a diferencia de las muestras de agua de llave. Por último, se mostró que la cantidad de agua filtrada, llenando el depósito del filtro dos veces al día, fue suficiente para el consumo de una unidad familiar con cinco personas el cual tiene un promedio de caudal de filtración de $1 \mathrm{~L}$ por hora aproximadamente. (Baide, 2011).

Lecciones aprendidas, retos y desafíos con el uso del filtro "Waterbackpack Paul" en Cundinamarca, Caldas, Cauca y Nariño, Colombia

En 56 familias de las comunidades de los indígenas wayuu en la Guajira, 200 familias de campesinos de Silvania-Cundinamarca-, 25 familias de Escuela Nevado del Ruiz en Villamaría -Caldas-, 20 familias de afrodescendientes de El Tajo -Cauca-, campesinos de El Encano -Nariño- y 77 familias de afrodescendientes de El Charco - Nariño-, se implementó la tecnología del filtro PAUL - ver Tabla 1 , sección D1-. Se trata de una unidad portátil de tratamiento de agua que utiliza una membrana de ultrafiltración que elimina el $99 \%$ de agentes contaminantes, con un caudal de filtración de 1200 L/día, desarrollada en el departamento de Ingeniería Sanitaria y Ambiental de la Universidad de Kassel Alemania -Desee-.

PAUL se basa en una membrana de ultrafiltración con celdas planas fabricadas de polietilsulfón -PESde tamaño poroso de $40 \mathrm{~nm}$, con la aplicación de la tecnología Ultra Low Pressure-Ultra Filtration Prozess - ULP-UF-. Las experiencias en campo con el filtro PAUL evidenciaron que puede ser usado para el tratamiento de agua potable en comunidades rurales por la alta remoción de patógenos, la robustez y a facilidad de operación y mantenimiento. La implementación de esta tecnología es eficiente puesto que tiene una durabilidad de diez años aproximadamente y en el país ha dado grandes beneficios para las comunidades en donde se encuentra. Actualmente se hacen estudios de adaptabilidad (Frechen, 2014).

\section{CONCLUSIONES}

1. El uso de filtros es una alternativa viable en las comunidades -que no tienen acceso a agua potable - en condiciones donde se tiene un deficiente o nulo sistema de acueducto o en situaciones de emergencia.

2. En los estudios se evidenció una variedad de filtros que son utilizados para satisfacer la necesidad de acceso a agua potable de las comunidades 
puesto que en la mayoría de los casos se probaron diferentes materiales de fácil acceso en los contextos locales -arcilla, contenedores plásticos, arenas y gravas, entre otros-.

3. Los filtros más utilizados fueron los de arcilla con baño de plata coloidal y los de arena lentos, pues son de accesibles tanto a nivel económico como a nivel de repuestos y mantenimiento, lo cual posibilita una alternativa para dar respuesta a las comunidades que no cuentan con acceso a agua potable o para mejorar las características del recurso hídrico.

Tabla 1. Sistemas de filtración utilizados actualmente

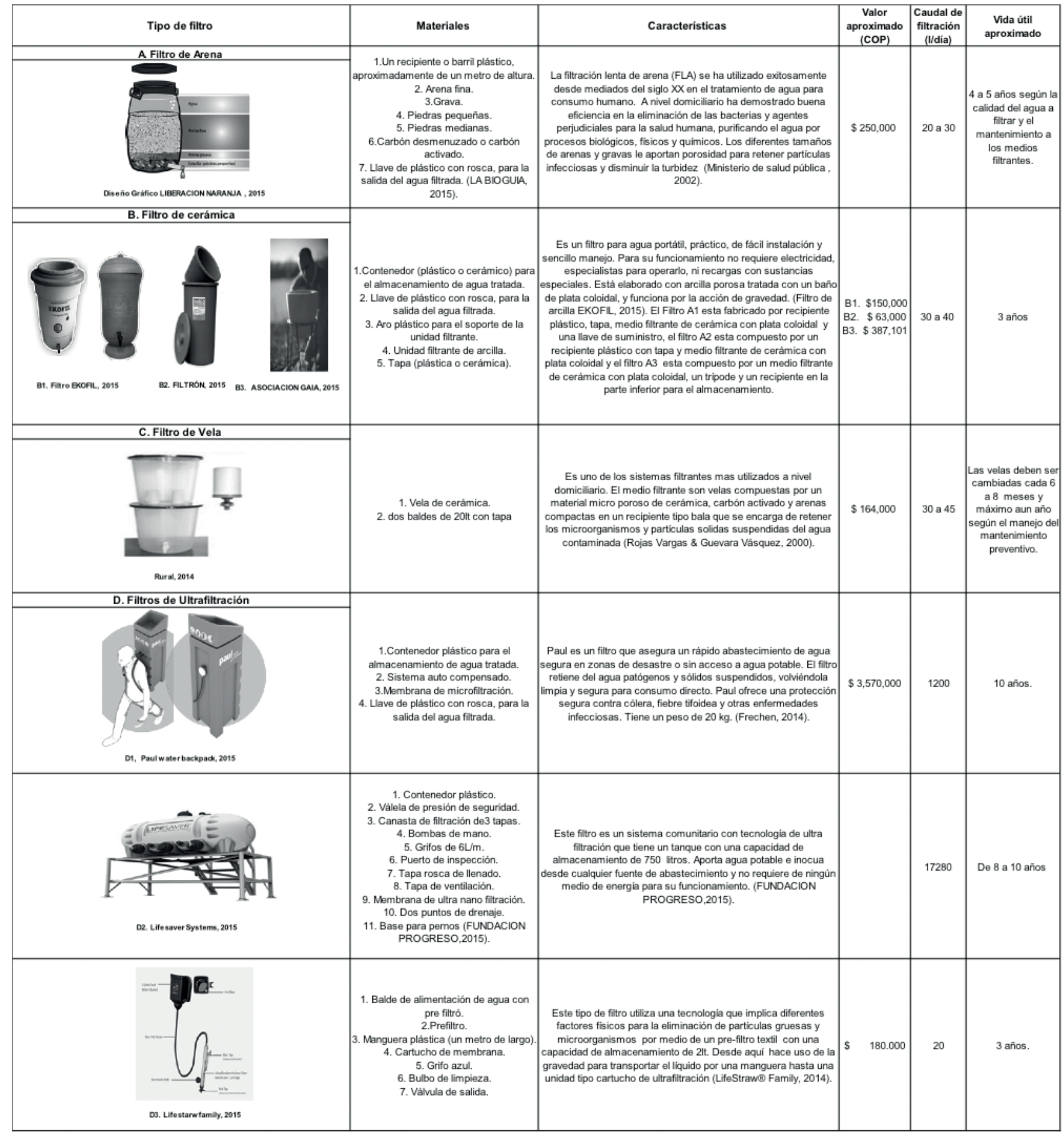

Fuente: material recopilado por los investigadores. 


\section{REFERENCIAS}

Baide, J. (2001). Prueba de la captación del filtro de cerámica impregnado con plata coloidal en el barrio El Ocotal de Guinope.

Barrientos Echegaray, H., Tello, J., Tito, C., y Palomino, M. (2009). Purificación de agua por medio de filtros de arena en la comunidad de KuyChiro. Cusco.

Burgos, D. (2006). Organización Panamericana de la Salud: Filtro de velas. Bogotá: Manual de construcción y mantenimiento.

Cavero, J. (2004). Mejoramiento de la calidad del agua de consumo en los caseríos de Tambogrande, Pirua distemización de la experiencia. Línea \& Punto SAC.

Defensoría del Pueblo (2006). Informe defensorial $N^{\circ} 39-B$.

Filtro de arcilla Ekofil. (2015). Recuperado de: http:// www.replacol.com

Frechen, J. (2014). Aplicación de la tecnología de filtración por membranas como solución para el abastecimiento de agua potable en comunidades rurales de países en desarrollo. Lecciones aprendidas con el uso del filtro «Waterbackpack PAUL». Revista Ambiental Agua, Aire Y Suelo, 3-7.

García, J. (1995). Estudio sanitario del agua filtración. Granada: Universidad de Granada.

Giordan, A., y Souchon, C. (1995). La educación ambiental: guía práctica. Sevilla: Díada.

Lesikar, B. (2000). Filtro de arena. Servicio de Extensión Agricola. Texas.

Masari, H. (2003). El agua como recurso. Revista Como Ves, 54, 10-12.

Navarro, J. (2008). Recursos hídricos y cambio climático. Cambioclimatico.org. Recuperado el 22 de septiembre de 2016, de http://www.cambioclimatico.org/content/recursos-hidricos

ONU. (2000). Indice de Desarrollo Humano. San José de Costa Rica: Agencia EfE.

Perdomo, C. (2014). Filtro de arena lento: Manual para el armado, instalación y monitoreo. Bogotá: Universidad Piloto de Colombia.

Pérez Vidal, A., Díaz, J., y González, G. (2014). Estudio comparativo de dos sistemas de filtración casera para el tratamiento de agua para consumo humano. Tunja: Universidad de Boyacá.

Ramírez, Plazas, J., Torres, C., Silva, J., Caicedo, L., \& González, M. (2011). A Systemic Framework to Develop Sustainable Engineering Solutions in Rural Communities in Colombia. Bogotá: Syst Pract Action Res.

RECA, WASH Cluster, Cruz Roja, unICEF. (2014). Seminario de intercambio de experiencias, tecnologías y metodologías en agua, saneamiento $e$ higiene en zonas vulnerables y en emergencias [en línea] Recuperado de: https:// www. humanitarianresponse.info/system/ files/documents/files/Informe\%20Seminario\%20Intercambio\%20de\%20Experiencias $\% 20$ ASH.pdf.

Rojas, R., y Guevara, S. (2000). Filtros de mesa. Lima.

Salud, O. (2005). Tecnologías para abastecimiento de agua. Lima: Centro Panamericano de Ingeniería Sanitaria y Ciencias del Ambiente.

UNAD. (2015). Diseño de plantas potabilizadoras. Recuperado de: http://datateca.unad.edu.co/ contenidos/358040/Contenido_en_linea_Diseno_de_Plantas_Potabilizadoras/index.html

Universidad de Manizales. (2015). Sistemas de tratamiento de aguas de la maestria en desarrolo sostenible y medio ambiente de la cátedra manejo integrado del agua. Manizales: Universidad de Manizales. 